\title{
Structure of Atomic Nuclei
}

$\mathrm{T}^{\mathrm{o}}$ 0 give a report of a discussion on a subject so complicated as the physics of the nucleus would be an arduous task at any time, but when it is an occasion for dealing with two new discoveries, it is a task the difficulty of which is sufficient excuse for any shortcomings. Not only did Dr. J. Chadwick describe his experiments leading to the identification of the neutron at the discussion on the subject at the Royal Society on April 28, but also Lord Rutherford reported that Dr. Cockcroft and Dr. Walton, working in the Cavendish Laboratory, had succeeded in effecting the artificial disintegration of several elements with protons from a high potential discharge tube.

The discussion was opened by Lord Rutherford in a characteristic address. Referring briefly to a number of different subjects, he showed convincingly how rapidly the subject of nuclear physics has extended in recent years. There is now a variety of lines of attack upon what he has appropriately termed the central problem of physics. Optical methods have been shown to lead to information about the masses of isotopes and the type of statistics they obey, while the details of the hyperfine structure give a direct measurement of the nuclear spin. Radioactivity has shown itself capable of revealing new details about the heaviest nuclei, and a close investigation of the $\alpha$-rays has thrown new light on the origin of the $\gamma$-rays. Quite apart from the information they yield about the nuclei from which they originate, the $\gamma$-rays and $a$-rays have proved as useful in recent years as in the past for investigating the fundamental laws governing high frequency radiation and high speed particles. A new type of absorption of electromagnetic radiation by the nucleus has been brought out by the work of Meitner, Hupfild, Tarrant, and Gray, which the last two workers believe to involve a true excitation of the nucleus.

In the more colloquial sense, however, it is in the field of artificial disintegration that the most exciting results have been obtained. A great deal of work has been carried out in different laboratories recently on the production and control of high voltages, and Cockcroft and Walton, working in the Cavendish Laboratory, have developed the technique of producing and using steady high potentials so high as 600,000 volts. They investigated the effect of bombarding lithium with protons of a few hundred thousand volts, and found the striking result that the lithium nucleus can absorb the proton and break up into two a-particles, each of which recoils with an energy corresponding to more than eight million volts (Nature, April 30, p. 649).

The experiments are simple in principle even if the technique is difficult. By means of a specially built discharge tube, protons are accelerated up to energies of several thousand volts. They are allowed to fall on a screen covered with lithium, to the side of which, and just outside the tube, is placed a zinc sulphide screen. A window covered with thin mica is arranged in the side of the tube, so that particles coming from the lithium can, if they have sufficient energy, reach the zinc sulphide screen. It was known from previous measurement that the protons themselves had not sufficient energy to penetrate the mica and reach the zinc sulphide screen, even if they were reflected from the lithium oxide without loss of energy. Bright scintillations were observed in the zinc sulphide as soon as the energy of the proton beam exceeded 120 thousand volts, and the numbers of these scintillations increased very rapidly as the voltage was raised. The particles causing these scintillations had a range in air of about $8 \mathrm{~cm}$., whereas the protons responsible for their emission could only penetrate about $1 \mathrm{~cm}$. This simple fact alone is a definite proof that disintegration involving a large emission of energy had occurred. The general character of the scintillations, coupled with the range of $8 \mathrm{~cm}$., suggested that these particles were a-particles, and this was borne out by the nature of the tracks obtained in an expansion apparatus. The process is therefore imagined to be

$$
\mathrm{Li}^{7}+\mathrm{H}^{1} \rightarrow 2 \mathrm{He}^{4} \text {. }
$$

From Aston's accurate measurements of the masses of these atoms, it follows that there would be a liberation of from 15 to 17 million electronvolts of energy in this process, in excellent agreement with the energy of $8 \times 10^{6}$ electron-volts which helium nuclei need in order to have a range of $8 \mathrm{~cm}$. While it therefore seems almost certain that this is a correct description of what is happening, Cockcroft and Walton were careful to emphasise that the nature of the high speed particle liberated in the disintegration had not yet been definitely established. Additional evidence will be provided if it is possible to show, as would follow from this hypothesis, that the particles always occur in pairs travelling in opposite directions.

The magnitude of the effect may be illustrated by the following figures. The effect becomes appreciable once the proton beam is accelerated with more than 120 thousand volts, and at 250 thousand volts one disintegration particle was obtained for about every $10^{9}$ protons hitting the lithium. The tube could be run at several microamperes, and since one microampere corresponds to about $10^{13}$ protons per second, it is clear that even with small solid angles there are plenty of particles to detect. Herein lies one of the important advantages of this new method. The yield of disintegration particles obtained even with the eight million volt particles from radioactive bodies is only of the order of one millionth, and it is a very powerful radioactive source which emits $2 \times 10^{9} \alpha$-particles per second, and those in all directions. It is easily seen that, despite the inefficiency of these low velocity protons, the actual number of disintegrations that can be effected is very much greater than with particles of radioactive origin, due to the large number of protons that can be used. 
In still another direction this discovery is of importance, since it gives a different type of nuclear disintegration. Using $a$-particles, it has been found that a proton is ejected and the $\alpha$-particle is captured, forming an atom of greater mass. If protons cause a disintegration, they must form an atom of smaller mass and lower in the periodic system.

Similar experiments have been tried with other elements, and it has been found that beryllium, boron, carbon, possibly nitrogen, fluorine and aluminium all give particles of characteristic range and of greater energy than the incident protons; oxygen and copper gave no effect. While disintegration occurred with both boron and aluminium with protons of energy so low as 150 thousand volts, with the other elements it was necessary to go to more than 300 thousand volts before noticeable effects were produced. It would therefore seem that the greater majority of the lighter elements can be disintegrated by bombardment with protons. While it is easy to speculate that processes such as

$$
\text { or } \begin{aligned}
\mathrm{Fl}^{19}+\mathrm{H} & =\mathrm{He}+\mathrm{O}^{16} \\
\text { (16 } & =\mathrm{He}+\mathrm{Mg}^{24}
\end{aligned}
$$

may occur, there is as yet no evidence about the nature of the particles except that which has been described in the case of lithium, and conclusions of any kind are mere guess-work until more information has been obtained.

Dr. Chadwick began by describing the results that have been obtained from investigating the collisions of $a$-particles with the nuclei of the lighter elements. Scattering experiments on one hand, and artificial disintegration experiments on the other, have combined to yield a wealth of information that could scarcely have been anticipated a few years ago. If these experiments of Chadwick and others are not diseussed here, it is not because of any lack of recognition of their fundamental importance and interest, but merely to come more quickly to Chadwick's discovery of the neutron.

Bothe and Becker first noticed that a very penetrating radiation was emitted when beryllium was bombarded with a-particles. Working with this beryllium 'radiation' and using an ionisation method, Joliot-Curie and Joliot found the surprising result that protons were ejected when it passed through materials containing hydrogen. If, as had always previously been supposed, this beryllium 'radiation' was of electromagnetic nature, the quantum would have to have an energy of $50 \times 10^{6}$ volts to account for the observed range, $30-40 \mathrm{~cm}$. in air, of the protons. In addition, it was particularly difficult to understand how the absorption process could occur so frequently.

Chadwick investigated this phenomenon, using the powerful valve counter methods which had been developed in the Cavendish Laboratory, and found some most surprising results. It appeared that this beryllium 'radiation' liberated particles from all light elements through which it passed, but if these processes were held to be due to interaction with some electromagnetic radiation, the quantum had to be $100 \times 10^{6}$ volts from the results with nitrogen, $150 \times 10^{6}$ volts from argon, whereas the value $50 \times 10^{6}$ volts was obtained from experiments with hydrogen. Chadwick realised that these difficulties disappeared if the beryllium ' radiation' were assumed to be of corpuscular nature, and then the particles detected by the valve counter would be atoms of the material radiated set in motion by collision with these corpuscles.

Now the beryllium 'radiation' is extremely penetrating; it loses less than one-half of its intensity in passing through one inch of lead, so that if it is corpuscular, then the corpuscles must be only very slightly affected by the atoms through which they pass. This can only mean that the field of influence of these corpuseles is limited to a minute space around them, and that they can pass close to an electron or nucleus without disturbing it or giving energy to it. A neutron, that is a proton and electron in close combination, would have just such properties, and, moreover, if its mass were about unity, and its velocity sufficiently high, it could set other atoms in motion on the rare occasions of direct collisions with the nuclei.

Important confirmation of the correctness of these general ideas was furnished by experiments in which the beryllium 'radiation' was passed through nitrogen contained in an expansion chamber. While no tracks were found corresponding to the neutron itself, clear photographs were obtained of nitrogen recoil atoms set in motion by the process already described. Chadwick has produced convincing evidence of the accuracy of this hypothesis by the following arguments based on his experiments. From measurements of the ranges, he determined the maximum velocities $u_{\mathrm{H}}$ and $u_{\mathrm{N}}$ which hydrogen and nitrogen nuclei could acquire by collision with a neutron. This evidently occurs in a head-on collision, so that if $m$ and $v$ denote the mass and velocity of the neutron, it follows from the conservation of energy and momentum that

$$
\begin{aligned}
& u_{\mathrm{H}}=\frac{2 m}{m+1} \cdot v \\
& u_{\mathrm{N}}=\frac{2 m}{m+14} \cdot v
\end{aligned}
$$

Inserting the experimental values, he obtained a value for $m$ slightly greater than unity, and a velocity about $3 \times 10^{9} \mathrm{~cm}$. per second. He obtained a far more accurate estimate of the mass of the neutron from a consideration of its origin. Chadwick and also Joliot-Curie and Joliot had discovered that neutrons are liberated from boron as well as from beryllium. In the former case the process is presumably

$$
\mathrm{B}^{11}+\mathrm{He}^{4} \rightarrow \mathrm{N}^{14}+n^{1}
$$

where $n^{1}$ denotes the neutron of mass about unity. Aston has made accurate measurements of the masses of boron, helium, and nitrogen, so that utilising the connexion between mass and energy given by the relativity theory, the energy balance of the process can be examined. It is found, taking into account the kinetic energy of the $\alpha$-particle, that the velocity of the neutron, obtained by the method already mentioned, can only be accounted for if the mass of the neutron lies between 1.005 and 1.007. Since the mass of a free hydrogen atom is 1.0078 , it follows that the energy of binding of 
the proton and electron is about one million volts. The combination of all this evidence can leave little doubt that the neutron really does exist and has been detected.

This discovery of a new kind of matter, a new element, is an outstanding event in the history of physics, and it is to be anticipated that important advances in our knowledge of the laws governing protons and electrons will be reached from investigation of its properties. Entirely new fields of speculation about the structure of atomic nuclei are opened up by the discovery that in at least two cases neutrons form part of the structure, but it is far too early to discuss matters of this kind.

Quite apart from the interest attached to the discovery of the neutron itself, it appears that it offers new possibilities of investigation in the field of artificial disintegration. Chadwick, in collaboration with Feather and Dee, has found that neutrons can also effect the disintegration of the nuclei with which they collide. This will constitute again another type of nuclear reaction, different from that produced either by $a$-particles or protons. For example, if it involved capture of the neutron and ejection of the proton, the resulting nucleus would have the same mass as before, but the atomic number would be lower by one.
There are important experimental advantages connected with the use of neutrons. The neutron is almost selective ; it produces no effect unless it makes an almost direct collision, and when this happens the probability is quite considerable that a disintegration will occur. For example, Feather has obtained 180 tracks in nitrogen, of which 30 were due to disintegration. Observation in the expansion chamber of disintegration by neutrons is much easier than the corresponding effect with a-particles, for since the neutron produces very little ionisation, a very large number of neutrons may be passed into the chamber per expansion without confusing the photographs. On the other hand, the interpretation of the disintegrations is more difficult, for neither the direction of the neutron nor its velocity at the moment of collision can be deduced from the photographs.

Important contributions to the discussion were made by many others, but they will not be referred. to now. It is excusable on this occasion if the well-established branches of the subject are asked to give way to two such promising newcomers. It is not intended to prejudge their ultimate importance, but only to conform to the interest which everyone will feel in these new discoveries.

C. D. Elifis.

\section{Oil and Petrol from Coal*}

By Prof. C. H. Lander, C.B.E.

$\mathrm{B}^{\mathrm{y}}$ $Y 1927$ the technical aspect of the hydrogenation of coal had reached so promising a stage that Imperial Chemical Industries, Ltd., thought it

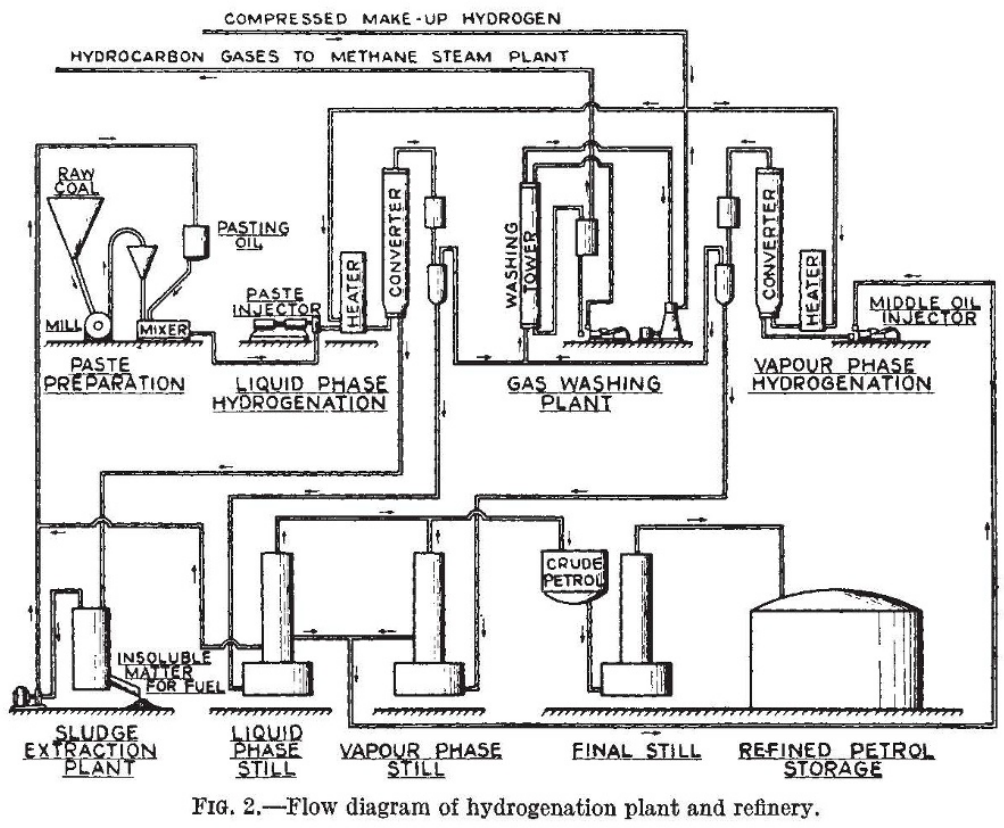

Billingham factory, where a full-scale plant capable of dealing with ten or fifteen tons of coal per day has since been developed. Fig. 2 shows the modified process as it is carried out at Billingham. The converters are now vertical, and are heated internally; thus the serious difficulties caused in the earlier plant, by the action of hydrogen upon metals at high temperatures, have been obviated. The material passes into the converters at a fairly high temperature, and the heat of the reaction, which is exothermic, is more than sufficient to maintain the process. A relatively thin paste is used, which can be injected into the converter at a pressure of 200-250 atmospheres, where it is treated with hydrogen at a temperature of $450^{\circ} \mathrm{C}$

The hydrocarbon vapours, the volatile fractions of oil, the water, ammonia, etc., are carried away at the top of the converter in the stream of excess hydrogen. The liquid products are condensed under pressure, and the permanent gases are passed on for oil scrubbing, after which they are recirculated through the plant. The hydrocarbons scrubbed out of the gas are passed to a 'methane-steam' plant for the production of worth while to explore independently the possibility of its commercial exploitation, and for this purpose a large research section was set up at its

* Continued from p. 641.

No. 3262, VoL. 129] 